\title{
Ultrafast Coupling between Light, Coherent Lattice Vibrations, and the Magnetic Structure of Semicovalent $\mathrm{LaMnO}_{3}$
}

\author{
S. Wall, ${ }^{1, *}$ D. Prabhakaran, ${ }^{1}$ A. T. Boothroyd,${ }^{1}$ and A. Cavalleri ${ }^{1,2}$ \\ ${ }^{1}$ Department of Physics, Clarendon Laboratory, University of Oxford, United Kingdom \\ ${ }^{2}$ Max Planck Group for Structural Dynamics, University of Hamburg, CFEL, Hamburg, Germany \\ (Received 15 March 2009; revised manuscript received 12 July 2009; published 27 August 2009)
}

\begin{abstract}
Coherent lattice vibrations are excited and probed with pulses of 10 fs duration in $\mathrm{LaMnO}_{3}$. The measured frequencies correspond to those of Jahn-Teller stretching and of out-of phase rotations of the oxygen octahedra. Surprisingly, the amplitude and damping rate of both modes exhibit a sharp discontinuity at the Néel temperature, highlighting nontrivial coupling between light, lattice, and magnetic structure. We explain this effect by applying the Goodenough-Kanamori rules to the excited state of $\mathrm{LaMnO}_{3}$, and note that charge transfer can invert the sign of the semicovalent exchange interaction, which in turn perturbs the equilibrium bond lengths.
\end{abstract}

DOI: 10.1103/PhysRevLett.103.097402

Optical excitation can induce large and ultrafast changes of the macroscopic electronic [1] and structural [2] properties of strongly correlated electron systems. In manganites, it has been shown that light can be used to switch between an insulating and a conducting state [3], sometimes resulting in the ultrafast formation of a ferromagnetic state [4]. These effects are similar to those achieved by magnetic fields [5] and open the possibility of optically controlled high speed switches and data storage devices. However, an understanding of the microscopic mechanism is lacking.

We investigated the response of $\mathrm{LaMnO}_{3}$, a material that retains many essential features and the complexity of manganites, while not exhibiting colossal magnetoresistance or a photoinduced phase transition, normally only found in doped compounds. In addition, difficulties associated with phase separation [6] are not present, and a clearer picture of the microscopic physics can be gained from optical measurements. Most experiments to date have measured the dynamics on the $100 \mathrm{fs}$ time scale [7], too slow to resolve the relevant lattice motions, which extend to frequencies near $20 \mathrm{THz}$. Here, we report measurements with $10 \mathrm{fs}$ pulses, which are capable of detecting coherent atomic motions over the whole vibrational spectrum.

$\mathrm{LaMnO}_{3}$ is a half-filled Mott insulator. Its cubic perovskite structure is shown in Fig. 1(a). The crystal field of the oxygen octahedra splits the fivefold degenerate $3 d$ levels into a tightly bound, threefold degenerate, $t_{2 g}$ level and a higher energy, twofold degenerate, $e_{g}$ level. As a result of Hund's coupling, the $\mathrm{Mn}^{3+}$ ions adopt the high-spin electronic configuration $t_{2 g \uparrow}^{3} e_{g \uparrow}$. At $T_{\mathrm{JT}} \approx 780 \mathrm{~K}$ a cooperative Jahn-Teller distortion of the oxygen octahedra within the $a b$ planes lifts the degeneracy of the $e_{g}$ levels and gives rise to a staggered orbital structure. Below $T_{N} \approx 140 \mathrm{~K}$, $\mathrm{LaMnO}_{3}$ undergoes a second order phase transition to an $A$-type antiferromagnet, in which the spins are ferromag-
PACS numbers: 78.47.J-, 63.20.kk, 71.70.Gm, 75.47.Lx

netically aligned in the $a b$ plane and antiferromagnetically along the $c$ axis.

The magnetic and structural properties of $\mathrm{LaMnO}_{3}$ can be understood with the Goodenough-Kanamori (GK) rules [8], which explain the sign of the superexchange interaction between neighboring manganese cations connected by an oxygen anion. According to the GK rules, the exchange integral, $J$, will be negative, i.e., prefer antiferromagnetic alignment, if the semicovalent bond is formed between two
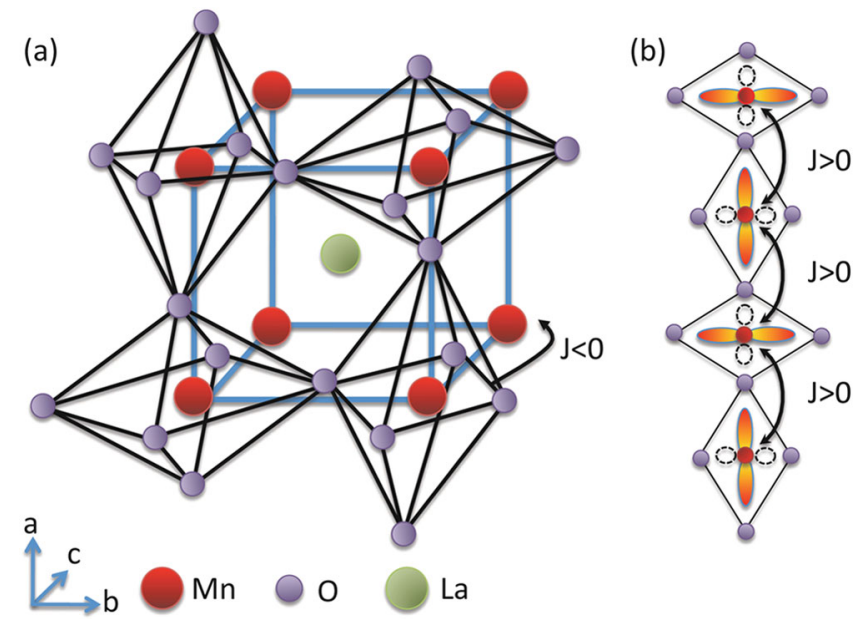

FIG. 1 (color online). (a) The unit cell of the cubic perovskite $\mathrm{LaMnO}_{3}$ with exaggerated rotations and Jahn-Teller distortions of the oxygen octahedra. The exchange interaction along the $c$ axis is dominated by semicovalent bonding between half-filled $t_{2 g}$ bonds resulting in an antiferromagnetic $(J<0)$ exchange. (b) Semicovalent bonding along the $a$ or $b$ axis. The shaded ellipses represent the occupied $d_{3 z^{2}-r^{2}}$ or $d_{3 y^{2}-r^{2}}$ orbitals of the $\mathrm{Mn}^{3+}$ ion, and the dashed circles represent the unoccupied $d_{x^{2}-y^{2}}$ or $d_{x^{2}-z^{2}}$ orbitals. Each occupied orbital overlaps with an unoccupied orbital via an oxygen anion resulting in a ferromagnetic $(J>0)$ exchange. 
half-filled orbitals or between two empty orbitals. This is because virtual hopping between two $e_{g}$ orbitals through the bridging oxygen $2 p$ orbitals is constrained by the Pauli exclusion principle.

When the bond is between a half-filled orbital and an empty orbital or between a filled orbital and a half-filled orbital, hopping is constrained by Hund's rule, and the exchange integral is positive, favoring ferromagnetic order. Furthermore, bonds that have an antiferromagnetic exchange interaction are shorter than those with a ferromagnetic interaction. According to Goodenough's description, magnetic bonds of this type are referred to as "semicovalent."

In $\mathrm{LaMnO}_{3}$, the Jahn-Teller-splitting of the $e_{g}$ levels gives rise to an occupied $d_{3 z^{2}-r^{2}}$ orbital and an unoccupied, $d_{x^{2}-y^{2}}$ orbital. Therefore, in the $a b$ plane, the $d_{3 z^{2}-r^{2}}$ orbitals will form semicovalent bonds, via the oxygen $2 p$ levels, with neighboring unoccupied $d_{x^{2}-y^{2}}$ levels as shown in Fig. 1(b). The application of the GK rules implies that $J>0$ in the $a b$ plane. Along the $c$ axis, bonds are formed between the oxygen-bridged $t_{2 g}$ levels. Because the $t_{2 g}$ levels are all half filled, the magnetic coupling is antiferromagnetic between the planes. The different exchange constants have been observed in the magnon dispersion curves measured within the $a b$ plane and along the $c$ axis, and the values $J_{a b}=0.83 \mathrm{meV}$ and $J_{c}=-0.58 \mathrm{meV}$ have been obtained [9].

Our experiments were performed on a large single crystal of $\mathrm{LaMnO}_{3}$, grown by the floating zone method [10] and cut in the ferromagnetic $a b$ plane. One kilohertz, sub10 fs optical pulses were generated by two noncollinear parametric amplifiers, with wavelength tunable between 490 to $700 \mathrm{~nm} . \mathrm{LaMnO}_{3}$ was the excitation with a central wavelength of $650 \mathrm{~nm}$, corresponding to the peak in the charge transfer excitation between neighboring $e_{g}$ levels [11]. The pump fluence at the sample surface was $6 \mathrm{~mJ} \mathrm{~cm}^{-2}$, above the threshold required to form the conducting state in other manganites [12]. The light was $s$ polarized, ensuring that the charge transfer excitation occurred along bonds within the $a b$ plane.

The time-dependent changes in reflectivity were measured from 80 to $300 \mathrm{~K}$ with $p$ polarization and a $600 \mathrm{~nm}$ central wavelength. Figure 2(a) shows measurements of the reflectivity change above $(200 \mathrm{~K})$ and below $(110 \mathrm{~K})$ the Néel temperature. The response of the system shows large amplitude coherent oscillations, superimposed on an exponential decay. The decay is fitted well with a double exponential of the form $\sum_{i=1,2} A_{i} e^{-t / \tau_{i}}$. We attribute the first term to rapid electron-electron thermalization, $\tau_{1}=$ 50-100 fs [the amplitude dependence on temperature is shown in Fig. 3(b)]. The slower, $\tau_{2}=1$ ps, term is attributed to electron-phonon thermalization.

Figure 2(b) shows the coherent oscillations after the electronic backgrounds have been subtracted. The Fourier transforms of the data are shown in Fig. 2(c).

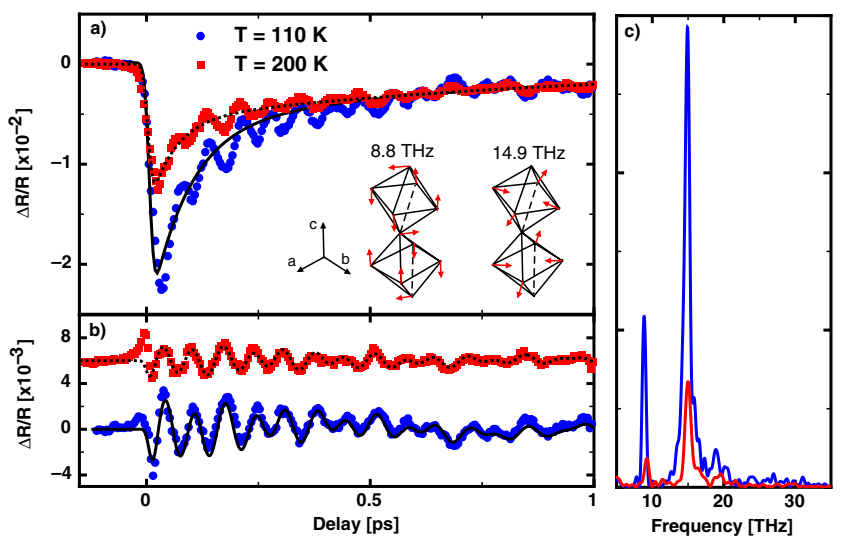

FIG. 2 (color online). (a) Time resolved pump-probe reflectivity transient taken at above $(200 \mathrm{~K}$, red squares) and below (110 K, blue circles) the Néel. The lines shows the fitted incoherent contribution to the relaxation. (b) The residual coherent oscillations after the decay has been subtracted. (c) The Fourier transform of the data, showing oscillations at 8.8 and 14.9 THz. The motion of the oxygen ions associated with each mode is indicated by the arrows in the diagram.

The two dominant oscillations have frequencies of $8.8 \mathrm{THz}\left(36.3 \mathrm{meV}, 293 \mathrm{~cm}^{-1}\right)$ and $14.9 \mathrm{THz}(61.5 \mathrm{meV}$, $497 \mathrm{~cm}^{-1}$ ) and correspond, with good agreement, to the values reported in Raman measurements reported by Iliev et al. [13] and Krüger et al. [14]. The atomic displacements of the modes are shown in the inset of Fig. 2(a) and correspond to the $A_{g}$ out-of-phase rotation $(8.8 \mathrm{THz})$ and $A_{g}$ Jahn-Teller stretching (14.9 THz) modes of the oxygen octahedra. A small amplitude is observed at $\sim 19 \mathrm{THz}$, corresponding to the polarization-dependent high-energy $B$ mode.

For each temperature, this signal was fitted with the sum of two damped oscillations of the form $\frac{\Delta R_{\text {osc }}}{R}=$ $-\sum_{i=1,2} B_{i} e^{-\Gamma_{i} t} \cos \left(2 \pi f_{i}\right)$, where $f_{1}$ and $f_{2}$ are fixed for all temperatures at the high $(14.9 \mathrm{THz})$ and low $(8.8 \mathrm{THz})$ frequency modes, respectively. $B_{i}$ is the oscillation amplitude, and $\Gamma_{i}$ is the damping rate of the mode. Both modes are found to be cosinelike, with no phase shift required for the fitting, indicating the modes are initiated by the displacive excitation of coherent phonons mechanism $[15,16]$.

The temperature dependence of the oscillation amplitudes is plotted in Fig. 3(a). The ratio between the two modes is constant over the entire temperature range. Surprisingly, the amplitudes of both modes increases sharply below the Néel temperature. This is not due to a change in absorption in the antiferromagnetic phase, as evidenced by the temperature-dependent amplitude of the electronic background in Fig. 3(b), which is dependent on the absorbed fluence, and shows a linear dependence with respect to changes in temperature. Therefore, the change in phonon amplitude can, most likely, be connected to a change in magnetoelastic coupling in the antiferromagnetic phase [17]. 

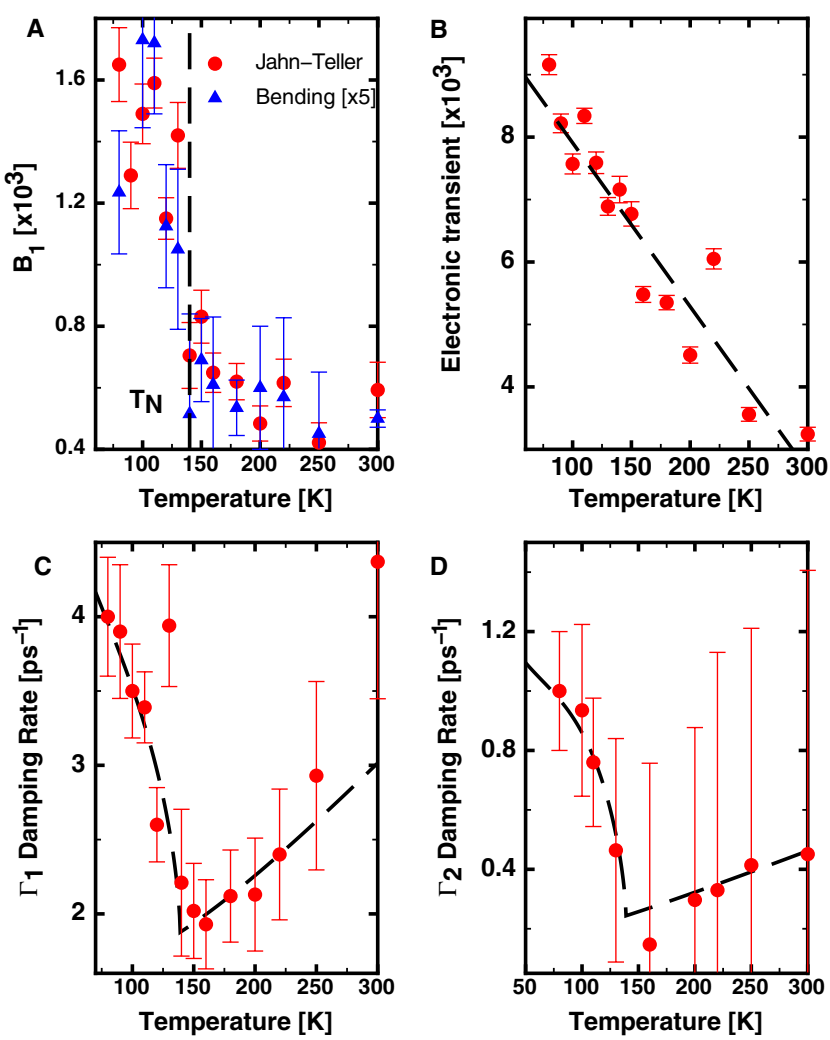

FIG. 3 (color online). (a) The oscillation amplitude of the 14.9 $\mathrm{THz}$ high frequency mode (red circles) and the $8.8 \mathrm{THz}$ low frequency mode (blue triangles, scaled by a factor of 5). Both modes have a similar temperature dependence, showing a significant enhancement in oscillation strength below the Néel temperature. (b) The amplitude of the electronic background, showing no effect at the Néel temperature. (c) Damping rate of the $14.9 \mathrm{THz}$ mode. (d) Damping rate of the $8.8 \mathrm{THz}$ mode. Above $T_{N}$ the behavior is described by anharmonic decay into acoustic phonon pairs. Below $T_{N}$ the scattering rate is increased due to coupling to magnons. Dashed black lines in (c) and (d) correspond to fits following the procedure in Ref. [19].

Figures 3(c) and 3(d) show the temperature dependence of the damping rates of the modes, which also show a similar anomaly. The damping rate is found to rapidly decrease as the temperature is reduced toward $T_{N}$, as expected for a decay channel dominated by scattering with phonons. Below $T_{N}$ the rate for both modes rises again, becoming progressively more pronounced as the antiferromagnetic phase is cooled.

We explain the increase in mode amplitude by considering how light excites the electronic structure in $\mathrm{LaMnO}_{3}$. Excitation at $1.9 \mathrm{eV}$ corresponds to charge transfer excitations between $\mathrm{Mn}$ ions of the form $2 \mathrm{Mn}^{3+}\left(t_{2 g \uparrow}^{3} e_{g \uparrow}\right) \rightarrow$ $\mathrm{Mn}^{+4}\left(t_{2 g \uparrow}^{3}\right)+\mathrm{Mn}^{2+}\left(t_{2 g \uparrow}^{3} e_{g \uparrow}^{2}\right)$. As a result, both Mn ions are no longer Jahn-Teller active and will prefer to relax the distortion. Therefore, the $14.9 \mathrm{THz}$ Jahn-Teller mode will be excited with large amplitude. The excitation mechanism of the slower, $8.8 \mathrm{THz}$, out-of-phase rotation mode has a more subtle explanation. The excited state is shown in Fig. 4(a) and corresponds to an in-plane excitation along the $a$ or $b$ axis. The $\mathrm{Mn}^{3+}-\mathrm{Mn}^{4+}$ bond in the excited state consists of two empty $e_{g}$ orbitals. Therefore, according to the GK rules, the exchange interaction will now become negative. Also, the $\mathrm{Mn}^{3+}-\mathrm{Mn}^{2+}$ bond consists of two half-filled $e_{g}$ levels, which also gives a $J<0$ exchange.

The change in sign of the exchange interaction will result in a force to reduce the Mn-O-Mn semicovalent bond length. This can be achieved by both the relaxation of the Jahn-Teller distortion and by reducing the bond angle to give a straighter bond. The latter motion is shown in Fig. 4(b) and maps directly onto the mode corresponding to the out-of-phase rotation of the oxygen octahedra. This description gives a qualitative explanation of the temperature dependence of the mode amplitude.

In the spirit of the displacive excitation of coherent phonons mechanism, in which the excited-state bond lengths change to minimize the potential energy of the system, we note that in our case two different processes contribute to the internal energy balance of the excited state. The first is a charge redistribution between $e_{g}$ orbitals, which suppresses Jahn-Teller activity. The second contribution comes from the modified, excited-state exchange interaction. The first channel is active at all temperatures up to $T_{\mathrm{JT}}=780 \mathrm{~K}$, whereas the second appears only below the Néel temperature.

The coupling of the magnetic degree of freedom to the lattice is further evidenced in the enhanced decay of the lattice vibrations below $T_{N}$. In a nonmagnetic system, the damping rate of an optic phonon is dominated by
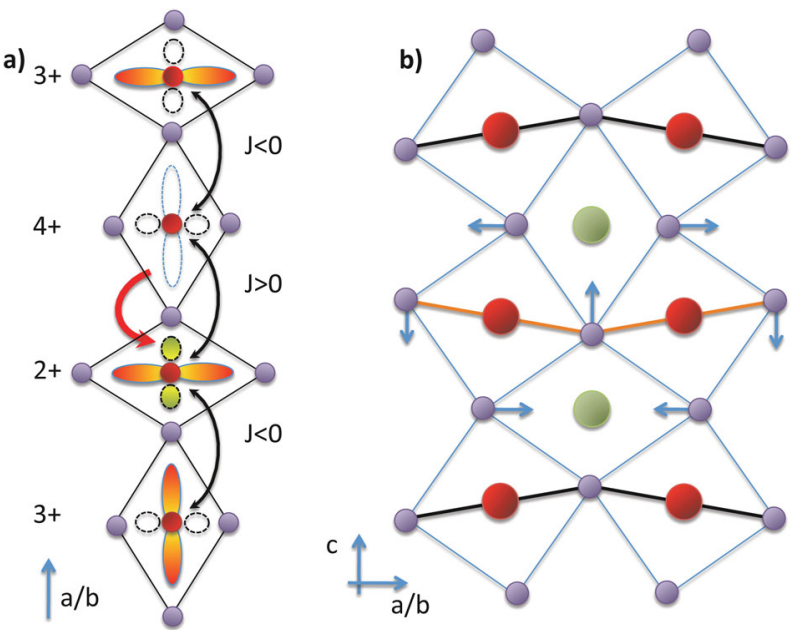

FIG. 4 (color online). (a) A state after a charge transfer excitation along the $a$ or $b$ axis and the effect on the exchange integral between Mn ions. (b) The photoexcited (highlighted) semicovalent bond experiences a change in sign of the exchange integral. As a result there is a force to reduce the $\mathrm{Mn}-\mathrm{O}-\mathrm{Mn}$ bond length. The induced displacements map onto the out-of-phase rotation of the $8.8 \mathrm{THz}$ mode. 
anharmonic decay into acoustic phonons [18], which increases with $T$. Above $T_{N}$ our data follow this temperature dependence well, with a fitted $T=0$ coupling strength of $\Gamma_{1}(T=0)=1.3 \mathrm{ps}^{-1}$. The discontinuity at $T_{N}$ indicates that an additional decay channel has become active, attributed here to magnon-phonon coupling. The temperature dependence below $T_{N}$ is well explained by coupling of the lattice to the magnetic degree of freedom. By following the procedure described in Ref. [19] [dashed black lines in Figs. 3(c) and 3(d)], a $T=0$ coupling strength of $\Gamma_{M}=$ $3.4 \mathrm{ps}^{-1}$ is found for the scattering of phonons by magnons.

To summarize, we have shown that coherently excited lattice motions in $\mathrm{LaMnO}_{3}$ exhibit anomalously strong correlation with the magnetic order. We explain this effect by applying the Goodenough-Kanamori rules to the excited state of the system, and note that charge transfer between neighboring $\mathrm{Mn}^{3+}$ sites can strongly perturb the local exchange interaction. These results highlight a new mechanism with which light can couple to the magnetic state of a complex solid, and provide a hypothesis to interpret photoinduced coupling between the charge, lattice, and spin. It also connects ultrafast elastic distortions to the magnetic degree of freedom and links to recent experiments where electronic and orbital phases were controlled by vibrational excitation [20,21]. The driving mechanisms discussed here expand, to the ultrafast time scale, many of the concepts used to describe the physics of electromagnons in multiferroics [22].

*simon.wall@physics.ox.ac.uk

[1] K. Miyano, T. Tanaka, Y. Tomioka, and Y. Tokura, Phys. Rev. Lett. 78, 4257 (1997).

[2] A. Cavalleri, C. Tóth, C. W. Siders, J. A. Squier, F. Ráksi, P. Forget, and J. C. Kieffer, Phys. Rev. Lett. 87, 237401 (2001).

[3] M. Fiebig, K. Miyano, Y. Tomioka, and Y. Tokura, Science 280, 1925 (1998).
[4] M. Matsubara, Y. Okimoto, T. Ogasawara, Y. Tomioka, H. Okamoto, and Y. Tokura, Phys. Rev. Lett. 99, 207401 (2007).

[5] Y. Tomioka, A. Asamitsu, H. Kuwahara, Y. Moritomo, and Y. Tokura, Phys. Rev. B 53, R1689 (1996).

[6] E. Dagotto, Nanoscale Phase Separation and Colossal Magnetoresistance (Springer, Berlin, 2003).

[7] H. Tamaru, K. Ishida, N. Ogawa, Y. Kubo, and K. Miyano, Phys. Rev. B 78, 075119 (2008).

[8] J. B. Goodenough, Phys. Rev. 100, 564 (1955).

[9] F. Moussa, M. Hennion, J. Rodriguez-Carvajal, H. Moudden, L. Pinsard, and A. Revcolevschi, Phys. Rev. B 54, 15149 (1996).

[10] D. Prabhakaran, A. I. Coldea, A. T. Boothroyd, and S. J. Blundell, J. Cryst. Growth 237, 806 (2002).

[11] N. N. Kovaleva, A. V. Boris, C. Bernhard, A. Kulakov, A. Pimenov, A. M. Balbashov, G. Khaliullin, and B. Keimer, Phys. Rev. Lett. 93, 147204 (2004).

[12] D. Polli, M. Rini, S. Wall, R. W. Schoenlein, Y. Tomioka, Y. Tokura, G. Cerullo, and A. Cavalleri, Nature Mater. 6, 643 (2007).

[13] M. N. Iliev, M. V. Abrashev, H.-G. Lee, V. N. Popov, Y. Y. Sun, C. Thomsen, R. L. Meng, and C. W. Chu, Phys. Rev. B 57, 2872 (1998).

[14] R. Krüger, B. Schulz, S. Naler, R. Rauer, D. Budelmann, J. Bäckström, K. H. Kim, S.-W. Cheong, V. Perebeinos, and M. Rübhausen, Phys. Rev. Lett. 92, 097203 (2004).

[15] T.E. Stevens, J. Kuhl, and R. Merlin, Phys. Rev. B 65, 144304 (2002).

[16] H. J. Zeiger, J. Vidal, T. K. Cheng, E. P. Ippen, G. Dresselhaus, and M. S. Dresselhaus, Phys. Rev. B 45, 768 (1992).

[17] T. Chatterji, P. F. Henry, and B. Ouladdiaf, Phys. Rev. B 77, 212403 (2008)

[18] F. Vallée, Phys. Rev. B 49, 2460 (1994).

[19] A. Melnikov, A. Povolotskiy, and U. Bovensiepen, Phys. Rev. Lett. 100, 247401 (2008).

[20] M. Rini, R. Tobey, N. Dean, J. Itatani, Y. Tomioka, Y. Tokura, R. W. Schoenlein, and A. Cavalleri, Nature (London) 449, 72 (2007).

[21] R. I. Tobey, D. Prabhakaran, A. T. Boothroyd, and A. Cavalleri, Phys. Rev. Lett. 101, 197404 (2008).

[22] A. A. Mukhin, V.Y. Ivanov, V.D. Travkin, A. M. Balbashov, A. Loidl, and A. Pimenov, Nature Phys. 2, 97 (2006). 